\title{
NOTAS E COMUNICAÇÕES \\ EFEITOS DO TAMANHO DA SEMENTE E DO RECIPIENTE NO CRESCIMENTO DE MUDAS DE CAMU-CAMU (Myrciaria dubia)
}

\author{
Kaoru YUYAMA ${ }^{1}$, Jhanssem Antônio Silva de SIQUEIRA ${ }^{2}$
}

RESUMO - O camu-camu (Myrciaria dubia (H.B.K.) McVaugh, Myrtaceae) é uma fruteira silvestre dos rios e lagos da Amazônia que está sendo domesticada para cultivo em solos de terra firme. O seu potencial econômico reside no seu alto teor de ácido ascórbico, que pode ser de até $2950 \mathrm{mg} / 100 \mathrm{~g}$ de polpa. O objetivo do presente trabalho foi avaliar o desenvolvimento das mudas de camu-camu provenientes de sementes de diferentes tamanhos, produzidas em diferentes volumes de substrato. O delineamento experimental foi de blocos casualizados, em esquema fatorial de $3 \times 4$, com quatro repetições. Os fatores foram tamanho da semente (pequena, média e grande, pesando 19,37 e $67 \mathrm{~g} / 100$ sementes, respectivamente) e tamanhos do recipiente (sacos plásticos pretos de $12 \times 23,16 \times 28,19 \times 21$ e $20,5 \times 33 \mathrm{~cm}$, com $750,1500,1750$ e $3500 \mathrm{~g}$, respectivamente). As mudas provenientes de sementes grandes e médias tiveram melhor desenvolvimento (altura de 53 e $46 \mathrm{~cm}$, respectivamente) e as mudas cultivadas em sacos de $19 \times 21 \mathrm{~cm}$ mostraram uma tendência de maior desenvolvimento (altura de $49 \mathrm{~cm}$ ), inclusive durante todo o período de cinco meses. Portanto, mudas de camu-camu com $30 \mathrm{~cm}$ de altura (pronto para o campo) podem ser obtidas em 60 a 70 dias após o transplante das plântulas provenientes de sementes grandes e médios e usando sacos de mudas com $19 \times 21 \mathrm{~cm}$.

Palavras-chave: Produção de mudas, volume de substrato, desenvolvimento.

\section{Effects of Seed Size and Nursery Bags on the Growth of Seedlings of Camu-camu} (Myrciaria dubia)

ABSTRACT - The camu-camu (Myrciaria dubia (H.B.K.) McVaugh, Myrtaceae) is a native Amazonian species of river and lake margins that is being domesticated for cultivation on the "terra firme". Its economic potential is its high ascorbic acid content, with $2,950 \mathrm{mg} / \mathrm{of}$ vit.C/ $100 \mathrm{~g}$ of pulp. Standards for production of certified seedlings are vague. The present study investigated the development of seedlings obtained from different size seeds and transplanted to different size bags. The experimental design was randomized blocks, with a $3 \times 4$ factorial, with four replications. The factors were: three sizes of seeds (small, medium and large, weighing 19,

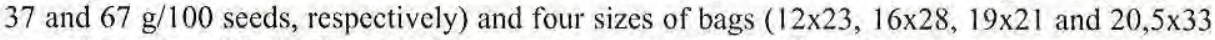
$\mathrm{cm}$, with $750,1500,1750$ and $3500 \mathrm{~g}$, respectively). The seedlings from large and medium size seeds grew faster (height 53 and $46 \mathrm{~cm}$, respectively) and those grown in the $19 \times 21 \mathrm{~cm}$ bags showed a tendency of faster growth (height $49 \mathrm{~cm}$ ) during all stages up to five months. Field ready seedlings of camu-camu can be obtained after 60 to 70 days when large and medium size seeds and $19 \times 21 \mathrm{~cm}$ bags are used.

Key-words: Seedling production, substrate volume, development.

Entre as diversas espécies de fruteiras existentes na Amazônia, o camu-camu (Myrciaria dubia (H.B.K.) McVaugh, Myrtaceae) vem despertando interesse em vários países, devido ao seu alto teor de ácido ascórbico (vitamina C), de $2950 \mathrm{mg}$ por $100 \mathrm{~g}$ de polpa, superior ao da maioria das plantas cultivadas (Andrade et al., 1991). O camu-camu

\footnotetext{
' Coordenação de Pesquisas em Ciências Agronômicas, Instituto Nacional de Pesquisas da Amazônia, Caixa Postal 478, 69.011-970, Manaus, AM, Brasil.

${ }^{2}$ Bolsista do CNPq/PIBIC-INPA.
} 
é um arbusto nativo das várzeas, margens de rios e lagos, adaptado às inundações periódicas, que sobrevive submerso na água por 4 a 5 meses durante o ano (Peters \& Vasques, 1986/87). Os frutos são consumidos pelos peixes, que são os principais dispersores da espécie, juntamente com a corrente d'água dos rios. Assim, ocorre ao longo dos rios e lagos de toda a região amazônica, onde se desenvolve em pequenas populações em locais em que a semente é depositada e encontra condições adequadas de temperatura, aeração e umidade.

O Instituto Nacional de Pesquisas da Amazônia (INPA) vem trabalhando com o camu-camu em terra firme desde 1978, testando sua adaptação fora do seu habitat natural. Existem normas e padrões para a produção de mudas fiscalizadas, elaboradas pela Comissão Estadual de Sementes e Mudas do Amazonas (CESM, 1996), mas estas ainda são vagas devido à falta de estudos específicos. No Peru, as plântulas são repicadas com 8 a $10 \mathrm{~cm}$ de altura e 6 a 8 folhas simples e, após 6 meses, estas plântulas devem alcançar 50 a 60 $\mathrm{cm}$ de altura e 7 a $8 \mathrm{~mm}$ de diâmetro (Ruiz \& Ramirez, s.d.); quando são repicadas com 10 folhas, depois de 6 a 8 meses devem alcançar 70 a $80 \mathrm{~cm}$ de altura e entre 6 e $9 \mathrm{~mm}$ de diâmetro a $30 \mathrm{~cm}$ do solo (Enciso \& Villachica, 1993; Villachica, 1996). Considerando que no Brasil inexistem informações sobre dois parâmetros importantes para produção de mudas de camucamu, este estudo teve como objetivo avaliar o desenvolvimento de plẩntulas no viveiro, variando-se o volume de substrato (tamanho do recipiente) e o tamanho da semente.

$O$ experimento foi realizado no viveiro do INPA, em Manaus, AM. O delineamento experimental foi de blocos casualizados, em esquema fatorial $3 \times 4$, com quatro repetições. Os fatores testados foram: tamanho da semente (pequena, média e grande, pesando 19,36 e $67 \mathrm{~g} / 100$ sementes, respectivamente) e tamanhos do recipiente (sacos plásticos pretos de $12 \times 23,16 \times 28,19 \times 21$ e $20,5 \times 33 \mathrm{~cm}$, com 750, 1500, 1750 e $3500 \mathrm{~g}$, respectivamente).

As sementes de camu-camu foram coletadas no dia 29 de março de 1997, em São Sebastião do Uatumã, $\mathrm{AM}$, nas margens do Rio Uatumã. $\mathrm{A}$ semeadura foi realizada no mês de abril de 1997, em uma caixa de madeira, utilizando serragem como substrato (camada de $15 \mathrm{~cm}$ ). As sementes de diferentes tamanhos foram semeadas separadamente e cobertas com uma camada de $1 \mathrm{~cm}$ de serragem. A germinação iniciou a partir de 15 dias da semeadura e prosseguiu por dois meses.

A repicagem foi realizada em 18 de agosto de 1997 (130 dias após a semeadura), selecionando as plântulas com altura aproximada de $9 \mathrm{~cm}$. O substrato utilizado para o enchimento dos sacos plásticos foi o horizonte $\mathrm{A}$ de um Latossolo Amarelo, de uma área cultivada com outras espécies, com a seguinte composição quimica: $\mathrm{pH}$ em $\mathrm{H}_{2} \mathrm{O}=5,5 ; \mathrm{Al}=4,9 \% ; \mathrm{K}=10 \mathrm{~g} /$ 
$\mathrm{kg} ; \mathrm{Ca}=2,9 ; \mathrm{Mg}=0,4 ; \mathrm{P}=6,9 \mathrm{ppm}$; $\mathrm{C}=1,6 \%$ ). Foram feitas sete medições de altura da muda e diâmetro do caule, dos 25 aos 153 dias após a repicagem.

$\mathrm{O}$ crescimento em altura das mudas provenientes de sementes grandes apresentou um tendência de superar o das de sementes médias e foi significativo para as sementes pequenas (Fig. 1); o crescimento no diâmetro do caule foi semelhante ao da altura das plantas $(r=0,99)$ (dados não apresentados). Em termos do tamanho de saco, foi observado uma tendência de diferença na altura das plantas somente a partir de três meses (Fig. 2), sendo o melhor crescimento no saco de $19 \times 21 \mathrm{~cm}$, seguido de $20,5 \times 33 \mathrm{~cm}$. Porém, este último é muito pesado e dificulta o transporte

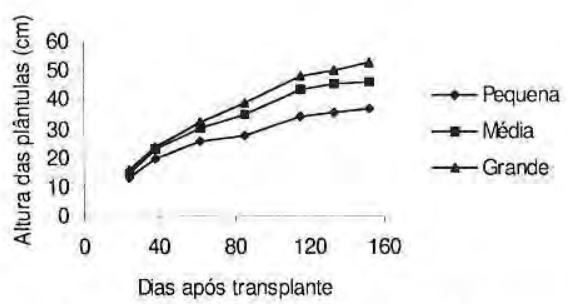

Figura 1. Crescimento em altura das plantas de camu-camu provenientes de diferentes tamanhos de sementes (DMS $=9,1 \mathrm{~cm}$ aos 153 dias, para $\mathrm{p}<5 \%$ ).

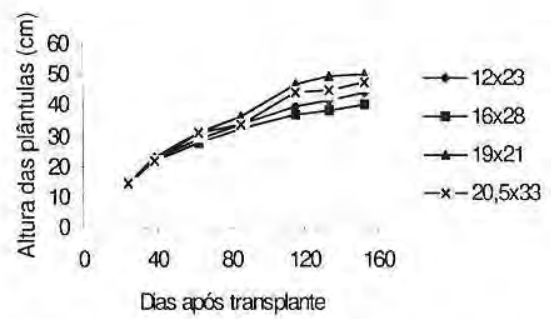

Figura 2. Crescimento em altura das plantas de camu-camu submetida a diferentes tamanhos de sacos. das mudas.

Não existe um tamanho mínimo de mudas para levar ao campo definitivo; existe uma recomendação para mudas enxertadas (quando igualar a grossura do porta enxerto e enxerto, e o ramo tenha no mínimo 60 cm; Villachica, 1996). Na Fazenda Yuricam, situado na rodovia AM-10 km 100 em Latossolo Amarelo, mudas seminíferas com $30 \mathrm{~cm}$ de altura foram plantadas no início de período chuvosa, tiveram um bom pegamento e $90 \%$ das plantas iniciaram a produção de frutos com menos de dois anos. Levando em consideração este tamanho $(30 \mathrm{~cm})$ para o plantio em campo definitivo, as mudas provenientes de sementes grandes e médias estariam aptas em 60 a 70 dias após o transplante nos sacos, enquanto mudas provenientes de sementes pequenas levarão mais 20-30 dias para chegar ao mesmo tamanho.

Concluiu-se que as sementes grandes originam plantas de crescimento mais rápido, apesar de não apresentar diferença significativa com as de sementes médias; o saco com as dimensões $19 \times 21 \mathrm{~cm}$ apresentam mudas com tendência de melhor desenvolvimento, também outrora nenhum dos tipos apresentarem diferenças significativas.

\section{Bibliografia citada}

Andrade, J.S.; Aragão, C.G.; Chávez F., W.B. 1991. Valor nutricional do camu-camu (Myrciaria dubia (H.B.K.) McVaugh) cultivado em terra firme da Amazônia Central, Revista Brasileira de Fruticultura, 13(3): 307-311. 
CESM. 1996. Normas e padrões para a produçăo de mudas fiscalizadas. Comissão Estadual de Sementes e Mudas/Delegacia Federal de Agricultura e Abastecimento no Amazonas/ Ministério da Agricultura e do Abastecimento, Manaus. 40p.

Enciso, R.; Villachica, H. 1993. Producción y manejo de plantas injertadas de camucamu (Myrciaria dubia) en vivero. Informe técnico n 25 , Instituto Nacional de Investigación Agraria, Lima. 20 p.

Peters, C.M.; Vasques, A. 1986/87. Estudos ecológicos de camu-camu (Myrciaria dubia). 1. Producción de frutos en poblaciones naturales. Acta Amazonica, 16/17: 161-174.

Ruiz, R.R.; Ramirez, I.J. s.d. Tecnologia del cultivo de camu-camu (Myrciaria dubia (H.B.K.) McVaugh) en la Amazonia Peruana. Instituto Nacional de Investigacion Agraria, Pucallpa. 45p.

Villachica, H. 1996. El cultivo del camu-camu (Myrciaria dubia (H.B.K.) McVaugh) en la Amazonia Peruana. Tratado de Cooperación Amazonica, Lima. 95p. 